\title{
On central limit theorems for IV-events
}

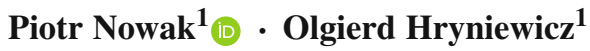

Published online: 17 July 2017

(C) The Author(s) 2017. This article is an open access publication

\begin{abstract}
Interval-valued fuzzy sets were introduced in 1970s as an extension of Zadeh's fuzzy sets. For intervalvalued fuzzy events, (IV-events for short) IV-probability theory has been developed. In this paper, we prove central limit theorems for triangular arrays of IV-observables within this theory. We prove the Lindeberg CLT and the Lyapunov CLT, assuming that IV-observables are not necessary identically distributed. We also prove the Feller theorem for null arrays of IV-observables. Furthermore, we present examples of applications of the aforementioned theorems. In particular, we study the convergence in distribution of scaled sums of identically distributed IV-observables.
\end{abstract}

Keywords Central limit theorem · IV-events · IV-probability

\section{Introduction}

The notion of a fuzzy set was introduced by L.A. Zadeh in 1965. The introduced notion of a fuzzy set is a powerful tool for studying different branches of science (in particular mathematics). It has been applied for introducing the fuzzy analogue of many notions existing for crisp sets. Some of these applications are due to Atanassov $(1999,2012)$, Couso et al. (2014), Dubois et al. (2005), Grattan-Guiness (1975), Tripathy and Ray (2012) and many others. Problems concern-

Communicated by A. Di Nola.

Piotr Nowak

Piotr.Nowak@ibspan.waw.pl

Olgierd Hryniewicz

Olgierd.Hryniewicz@ibspan.waw.pl

1 Systems Research Institute, Polish Academy of Sciences, ul. Newelska 6, 01-447 Warszawa, Poland ing convergence of series and sequences of fuzzy numbers have been considered, i.a., by Tripathy and Das (2012), Tripathy and Sarma (2012), and Tripathy et al. (2012).

The classical Kolmogorov's theory of probability is a commonly used mathematical model of randomness. It has been described in nearly all textbooks on probability and is well known both for scientists and practitioners dealing with random phenomena. However, there exist other approaches to model such phenomena proposed by mathematicians, physicists and philosophers (see, e.g. the book of Fine 1973). One of them, the Boolean algebraic probability theory, was proposed for the case of quantum systems. This approach is based on fundamental works of great mathematicians: C. Carathéodory, G. Birkhoff, and J. von Neumann. In their works (see, Birkhoff and von Neumann 1936 and Carathéodory 1956), they considered states and observables of a quantum system as counterparts of probability and random variables in the Kolmogorov theory of probability. The concepts of Carathéodory, Birkhoff, and von Neumann were further developed by Gudder (1979), Pták and Pulmannová (1989), Riečan and Neubrunn (1997), Varadarajan (1968), where quantum logics were considered as orthomodular posets.

In many practical applications, randomness is not the only source of uncertainty. The second such source is imprecision nowadays usually modelled by Zadeh's fuzzy sets. When uncertain phenomena of interest are both random and imprecise, the concept of a fuzzy random variable can be applied. There exist many definitions of the fuzzy random variable which have different interpretations (see, e.g. Kruse and Meyer 1987; Kwakernaak 1978; Liu and Liu 2003; Puri and Ralescu 1986; Couso et al. 2014). According to the first, introduced by Kwakernaak (1978), the fuzzy random variable can be interpreted as fuzzy perception of an original crisp random variable. According to this interpretation, the 
fuzzy random variable is a (disjunctive) fuzzy set of classical random variables and is described by a fuzzy set of classical probability distributions. This interpretation is nowadays called epistemic and allows to generalize in a relatively easy way the classical concepts of probability and mathematical statistics. Another definition was proposed by Puri and Ralescu (1986). According to their definition, to describe the fuzzy random variable $\sigma$-algebras of fuzzy sets are used. Thus, it is a classical random variable with values belonging to a set of functions. This interpretation of the fuzzy random variable understood in the sense of Puri and Ralescu is nowadays called ontic, and its theoretical foundations can be regarded as the generalizations of theory of random sets. For more information about different definitions and interpretations of fuzzy random variables, the reader is encouraged to read an excellent textbook by Couso, Dubois and Sánchez (2014), or the paper by Dubois and Prade (2012).

Fuzzy sets, according to Zadeh himself, were introduced in order to provide a precise mathematical description (i.e. to 'precisiate') of imprecise notions, described, e.g. using human's plain language. However, from a practical point of view non-random imprecision may have different, sometimes subtle, interpretations. Therefore, many different generalizations of fuzzy sets have been proposed. One of such generalizations, which nowadays has become quite popular, is the theory of IF-sets introduced by Atanassov (see Atanassov 1999, 2012 and references therein) in the 1980s. Another one is the theory of interval-valued fuzzy sets (IVF-sets), introduced independently (in the same year!) by four authors: Grattan-Guiness (1975), Jahn (1975), Sambuc (1975), and Zadeh (1975). In this case, the generalization of fuzzy sets consists in the considering the values of the membership function as uncertain intervals (see, e.g. Zadeh 1975 and Türkşen 1986). The mutual relationship between these two models has been studied by many authors who have shown their formal equivalence (see, Deschrijver and Kerre 2003 and Dubois et al. 2005). It is worth noticing that IVF-sets are also equivalent to grey sets introduced by Deng (1989) very popular in East Asia (see Deschrijver and Kerre 2003).

The majority of results about the fuzzy generalizations of the probability have been related to the classical Kolmogorov's definition of probability. However, fuzzy models of quantum mechanics have also been studied recently. One should mention the theories of F-quantum spaces and fuzzy quantum logics (for details see Riečan and Neubrunn 1997 and references therein). The fuzzy quantum logic of all measurable functions with values in the interval $[0,1]$ is an example of MV-algebra, introduced by Chang (1958). Fundamentals and the most important theorems of MV-algebraic probability theory, including the central limit theorem, can be found in Nowak and Hryniewicz (2015), Riečan (1999, 2000), Riečan and Mundici (2002), Riečan and Neubrunn
(1997). In the area of fuzzy sets, the MV-algebraic probability theory was also applied in the Atanassov's IF-sets setting. This application let Riečan (2007b) to develop the probability theory for IF-events. Riečan (2007b) proved the central limit theorem (CLT) for independent, identically distributed IF-observables and M-observables. Other results of probability theories concerning IF-events can be found, e.g. in Ciungu and Riečan (2010), Lendelová (2006), Lendelová and Petrovičová (2006), Renčová (2010), Riečan (2004, 2006a, b, 2007a). Generalized versions of central limit theorems within the IF-probability theory and Mprobability theory for non-identically distributed observables were proved in the recent paper by Nowak and Hryniewicz (2016).

In contrast to the probability theory of IF-sets, its counterpart for the probability theory of IVF-sets is much less developed. Some interesting results concerning the probability theory of IVF-sets have been published by Riečan and Král' (2010), Kuková (2011), and Samuelčík and Hollá (2013). The aim of this paper is to fill the gap between the well-developed theory of probability for IF-sets and the theory of probability for IVF-sets. It is devoted to central limit theorems within the IV-probability theory, i.e. the probability theory for IV-events, which involves the Łukasiewicz connectives between IVF-sets. Analysing the limit behaviour of the row sums of triangular arrays of independent IV-observables, we prove the Lindeberg CLT and the Lyapunov CLT as well as the Feller theorem, which is a converse of the Lindeberg CLT. We use a proving technique based on MV-algebraic probability theory from Nowak and Hryniewicz (2015). We additionally present three examples of applications of our theorems for sequences and arrays of IV-observables with convergent scaled sums or row sums. The first example is general and concerns a sequence of independent IV-observables with the same distributions. We prove an appropriate theorem for such a sequence and we apply it to the case corresponding to the classical de MoivreLaplace theorem. In the second and the third example, we apply the Lindeberg CLT and the Lyapunov CLT. In the last two examples, we use a special form of the IV-probability, basing on a modified notion of the probability of IF-events, considered by Szmidt and Kacprzyk (1999a), Szmidt and Kacprzyk (1999b), Grzegorzewski and Mrówka (2002) and generalized by Nowak (2003, 2004a, b).

Despite formal similarity between IF-sets and IVF-sets, the semantics used for their interpretation is different. Therefore, the results obtained in this paper may be useful for those practitioners for whom the semantics of IVF-sets is better understandable than the semantics of IF-sets. These results may be used in the development of statistical methods for IFV-sets which, as for now, are practically non-existent. In the development of such methods, one can use approaches, already developed for random fuzzy sets, and described in an 
overview paper by Gil and Hryniewicz (2009) or in a recent paper by Blanco-Fernández et al. (2014).

The paper is organized as follows. In Sect. 2, we introduce some elements of the theories of MV-algebras and MVprobability. Section 3 contains our main results, including the Lindeberg CLT, Lyapunov's CLT, and the Feller theorem for IV-observables. In Sect. 4, we analyse examples of applications of the limit theorems proved in Sect. 3. The last section is dedicated to conclusions.

\section{Basic notions and facts concerning the MV-algebraic probability theory}

Let $n \in \mathbb{N}$, where $\mathbb{N}$ is the set of all positive integers. We denote by $\mathbb{N}[n]$ and $\mathcal{B}\left(\mathbb{R}^{n}\right)$ the set $\{1,2, \ldots, n\}$ and the $\sigma$ algebra of Borel subsets of $\mathbb{R}^{n}$, respectively.

We will use following theorem (see Billingsley 1986, Theorem 16.12) concerning the change of variable for integrals.

Let $(X, \mathcal{X})$ and $\left(X^{\prime}, \mathcal{X}^{\prime}\right)$ be measurable spaces. Let $T$ : $X \rightarrow X^{\prime}$ be an $\mathcal{X} / \mathcal{X}^{\prime}$ measurable function, i.e. $T^{-1}\left(A^{\prime}\right) \in$ $\mathcal{X}$ for each $A^{\prime} \in \mathcal{X}^{\prime}$. For a measure $\mu$ on $\mathcal{X}$ we define a measure $\mu T^{-1}$ on $\mathcal{X}^{\prime}$ given by

$\mu T^{-1}\left(A^{\prime}\right)=\mu\left(T^{-1}\left(A^{\prime}\right)\right), \quad A^{\prime} \in \mathcal{X}^{\prime}$.

Theorem 1 Let $f: X^{\prime} \rightarrow \mathbb{R}$ be an $\mathcal{X}^{\prime}$-measurable function. If $f$ is non-negative, then

$\int_{X} f(T x) \mu(\mathrm{d} x)=\int_{X^{\prime}} f\left(x^{\prime}\right) \mu T^{-1}\left(\mathrm{~d} x^{\prime}\right)$.

A function $f$ (not necessarily non-negative) is integrable with respect to $\mu T^{-1}$ if and only if $f T$ is integrable with respect to $\mu$, in which case (1) and

$\int_{T^{-1}\left(A^{\prime}\right)} f(T x) \mu(\mathrm{d} x)=\int_{A^{\prime}} f\left(x^{\prime}\right) \mu T^{-1}\left(\mathrm{~d} x^{\prime}\right)$,

where $A^{\prime} \in \mathcal{X}^{\prime}$, hold. Moreover, for any non-negative $f$, the identity (2) always holds.

MV-algebras are considered as non-commutative generalizations of Boolean algebras. The fundamentals of the theory of MV-algebras were discussed, e.g. by Cignoli et al. (2000) and Mundici (1986). We present only selected elements of the theory of MV-algebras and the MV-algebraic probability theory from Riečan and Mundici (2002) and Nowak and Hryniewicz (2015) with minor modifications.

Definition 1 An algebra $(M, 0,1, \neg, \oplus, \odot)$, where $M$ is a non-empty set, the operation $\oplus$ is associative and commutative with 0 as the neutral element,

$\neg 0=1, \neg 1=0$, and for arbitrary $x, y \in M$

$$
\begin{aligned}
x \oplus 1 & =1, \\
y \oplus \neg(y \oplus \neg x) & =x \oplus \neg(x \oplus \neg y), \\
x \odot y & =\neg(\neg x \oplus \neg y),
\end{aligned}
$$

is called an $M V$-algebra.

In an MV-algebra $(M, 0,1, \neg, \oplus, \odot)$ the relation $\leq$ given by the condition

$x \leq y \Leftrightarrow x \odot \neg y=0, x, y \in M$

defines a partial order.

The distributive lattice $(M, \vee, \wedge)$ with least element 0 and greatest element 1 , where

$x \vee y=\neg(\neg x \oplus y) \oplus y$

and

$x \wedge y=\neg(\neg x \vee \neg y)$,

for $x, y \in M$, is called the underlying lattice of $M$.

Definition 2 We call an MV-algebra $M \sigma$ - complete (complete) if every sequence (non-empty family, respectively) of elements of $M$ has the supremum in $M$.

We will use the following notations. Let $\left\{A_{n}\right\}_{n=1}^{\infty}$ be a sequence of subsets of a set $X$. Then

$A_{n} \nearrow A$ iff $A_{1} \subseteq A_{2} \subseteq \ldots$ and $\bigcup_{n}^{\infty} A_{n}=A$

For a sequence $\left\{x_{n}\right\}_{n=1}^{\infty}$ of real numbers,

$x_{n} \nearrow x$ iff $x_{1} \leq x_{2} \leq \ldots$ and $x=\sup _{i} x_{i}$

Additionally, for a sequence $\left\{b_{n}\right\}_{n=1}^{\infty}$ of elements of an MValgebra $M$

$b_{n} \nearrow b$ iff $b_{1} \leq b_{2} \leq \ldots$ and $\quad b=\sup _{i} b_{i}$

with respect to the underlying order of $M$.

Within the MV-algebraic probability theory the notions of state and observable were introduced, by abstracting the properties of probability measure and classical random variable.

Definition 3 Let $M$ be a $\sigma$-complete MV-algebra. A state on $M$ is a function $m: M \rightarrow[0,1]$ fulfilling the following conditions for arbitrary $a, b, c \in M$ and $\left\{a_{n}\right\}_{n=1}^{\infty} \subset M$ :

(1) $m(1)=1$ 
(2) if $b \odot c=0$, then $m(b \oplus c)=m(b)+m(c)$;

(3) if $a_{n} \nearrow a$, then $m\left(a_{n}\right) \nearrow m(a)$.

We call a state $m$ faithful if $m(x) \neq 0$ for each nonzero element $x$ of $M$.

Apart from the defined above notion of state, its additive counterpart, for which $\sigma$-additivity is not assumed, is also considered in the literature (see Nowak and Hryniewicz 2015 for more details).

Definition 4 A pair $(M, m)$ consisting of a $\sigma$ - complete MValgebra $M$ and a faithful state $m$ on $M$ is called a probability $M V$-algebra.

It was proved that every probability MV-algebra is complete (see Mundici 2011, Theorem 13.8).

Definition 5 Let $M$ be a $\sigma$-complete MV-algebra. An $n$ dimensional observable of $M$ is a function $x: \mathcal{B}\left(\mathbb{R}^{n}\right) \rightarrow M$ fulfilling the following conditions:

(1) $x\left(\mathbb{R}^{n}\right)=1$;

(2) $x(A) \odot x(B)=0$ and $x(A \cup B)=x(A) \oplus x(B)$ for arbitrary $A, B \in \mathcal{B}\left(\mathbb{R}^{n}\right)$ such that $A \cap B=\varnothing$;

(3) for arbitrary $A, A_{1}, A_{2}, \ldots \in \mathcal{B}\left(\mathbb{R}^{n}\right)$ if $A_{n} \nearrow A$, then $x\left(A_{n}\right) \nearrow x(A)$.

Theorem 2 Let $M$ be a $\sigma$-complete $M V$-algebra, $x$ : $\mathcal{B}\left(\mathbb{R}^{n}\right) \rightarrow M$ be an $n$-dimensional observable, and $m$ be a state on $M$. Then the function $m_{x}: \mathcal{B}\left(\mathbb{R}^{n}\right) \rightarrow[0,1]$ given by

$m_{x}(A)=(m \circ x)(A)=m(x(A)), \quad A \in \mathcal{B}\left(\mathbb{R}^{n}\right)$

is a probability measure on $\mathcal{B}\left(\mathbb{R}^{n}\right)$.

For the proof of Theorem 2 we refer the reader to Nowak and Hryniewicz (2015).

Definition 6 Let $(M, m)$ be a probability MV-algebra. An observable $x: \mathcal{B}(\mathbb{R}) \rightarrow M$ of $M$ is said to be integrable in $(M, m)$ if the expectation $\mathbb{E}(x)=\int_{\mathbb{R}} t m_{x}(\mathrm{~d} t)$ exists. We say that $x$ is square-integrable in $(M, m)$ if $\int_{\mathbb{R}} t^{2} m_{x}(\mathrm{~d} t)$ exists. If $x$ is square-integrable in $(M, m)$, then its variance exists and is given by

$$
\begin{aligned}
\mathbb{D}^{2}(x) & =\int_{\mathbb{R}} t^{2} m_{x}(\mathrm{~d} t)-(\mathbb{E}(x))^{2} \\
& =\int_{\mathbb{R}}(t-\mathbb{E}(x))^{2} m_{x}(\mathrm{~d} t) .
\end{aligned}
$$

We denote by $L_{m}^{1}\left(L_{m}^{2}\right)$ the space of observables $x$ : $\mathcal{B}(\mathbb{R}) \rightarrow M$ integrable (square-integrable, respectively) in $(M, m)$. More generally, we write $x \in L_{m}^{p}$ for $p \geq 1$ if $\int_{\mathbb{R}}|t|^{p} m_{x}(\mathrm{~d} t)<\infty$.
Definition 7 Let $(M, m)$ be a probability MV-algebra. Observables $x_{1}, x_{2}, \ldots, x_{n}$ of $M$ are said to be independent (with respect to $m$ ) if there exists an $n$-dimensional observable $h: \mathcal{B}\left(\mathbb{R}^{n}\right) \rightarrow M$ such that for all $C_{1}, C_{2}, \ldots, C_{n} \in \mathcal{B}(\mathbb{R})$

$$
\begin{aligned}
m & \left(h\left(C_{1} \times C_{2} \times \cdots \times C_{n}\right)\right) \\
& =m\left(x_{1}\left(C_{1}\right)\right) \cdot m\left(x_{2}\left(C_{2}\right)\right) \cdot \ldots \cdot m\left(x_{n}\left(C_{n}\right)\right) \\
& =m_{x_{1}}\left(C_{1}\right) \cdot m_{x_{2}}\left(C_{2}\right) \cdot \ldots \cdot m_{x_{n}}\left(C_{n}\right) .
\end{aligned}
$$

Remark 1 Assume that $x_{1}, x_{2}, \ldots, x_{n}: \mathcal{B}(\mathbb{R}) \rightarrow M$ are independent observables in a probability $\mathrm{MV}$-algebra $(M, m)$ and $h: \mathcal{B}\left(\mathbb{R}^{n}\right) \rightarrow M$ is their joint observable. Then for any Borel measurable function $g: \mathbb{R}^{n} \rightarrow \mathbb{R}$

$g\left(x_{1}, x_{2}, \ldots, x_{n}\right)=h \circ g^{-1}$

is an observable.

We fix a sequence $\left\{k_{n}\right\}_{n \in \mathbb{N}}$ of positive integers such that $\lim _{n \rightarrow \infty} k_{n}=\infty$ and a sequence of probability MValgebras $\left\{\left(M_{(n)}, m_{(n)}\right)\right\}_{n \in \mathbb{N}}$. For each $n \in \mathbb{N}$ and arbitrary observable $x: \mathcal{B}(\mathbb{R}) \rightarrow M_{(n)}$ belonging to $L_{m_{(n)}}^{2}$ we denote by $\mathbb{E}_{(n)}(x)$ the expected value of $x$ and by $\mathbb{D}_{(n)}^{2}(x)$ the variance of $x$ with respect to $m_{(n)}$. Furthermore, for each $x: \mathcal{B}(\mathbb{R}) \rightarrow M_{(n)}$ belonging to $L_{m_{(n)}}^{2}$ and $\varepsilon, s>0$ we use the symbol $l_{n}^{x}(\varepsilon, s)$ to denote the $\mathbb{R}$-valued function of the form

$l_{n}^{x}(\varepsilon, s)=\mathbb{E}_{(n)}\left(\left(x-\mathbb{E}_{(n)}(x)\right)^{2} I_{\left|x-\mathbb{E}_{(n)}(x)\right|>\varepsilon s}\right)$.

Definition 8 Let us assume that for each $n \in \mathbb{N}\left\{x_{n 1}, x_{n 2}\right.$, $\left.\ldots, x_{n k_{n}}\right\}$ is a sequence of independent (with respect to $m_{(n)}$ ) observables of the MV-algebra $M_{(n)}$. We call $\left\{x_{n j}\right\}_{n \in \mathbb{N}, j \in \mathbb{N}\left[k_{n}\right]}$ a triangular array of independent observables (TA for short).

Definition 9 Let $\left\{x_{n j}\right\}_{n \in \mathbb{N}, j \in \mathbb{N}\left[k_{n}\right]}$ be a TA such that for each $n \in \mathbb{N}$

$x_{n j} \in L_{m_{(n)}}^{2}, \quad j \in \mathbb{N}\left[k_{n}\right], \quad s_{n}^{2}=\sum_{j=1}^{k_{n}} \mathbb{D}_{(n)}^{2}\left(x_{n j}\right) \in(0, \infty)$.

Then $\left\{x_{n j}\right\}_{n \in \mathbb{N}, j \in \mathbb{N}\left[k_{n}\right]}$ is said to satisfy the Lindeberg condition if for arbitrary $\varepsilon>0$

$L_{n}(\varepsilon)=\frac{1}{s_{n}^{2}} \sum_{j=1}^{k_{n}} l_{n}^{x_{n j}}\left(\varepsilon, s_{n}\right) \rightarrow 0$, as $n \rightarrow \infty$.

Definition 10 A TA $\left\{x_{n j}\right\}_{n \in \mathbb{N}, j \in \mathbb{N}\left[k_{n}\right]}$ fulfilling (3) is said to satisfy the Lyapunov condition if there exists $\delta>0$ such that 
$\frac{1}{s_{n}^{2+\delta}} \sum_{j=1}^{k_{n}} \mathbb{E}_{(n)}\left(\left|x_{n j}-\mathbb{E}_{(n)}\left(x_{n j}\right)\right|^{2+\delta}\right) \rightarrow 0$, as $n \rightarrow \infty$

Definition 11 Let $\left\{\left(M_{(n)}, m_{(n)}\right)\right\}_{n \in \mathbb{N}}$ be a sequence of probability MV-algebras. A sequence of observables $\left\{x_{n}: \mathcal{B}(\mathbb{R})\right.$ $\left.\rightarrow M_{(n)}\right\}_{n \in \mathbb{N}}$ is convergent in distribution to a function $F: \mathbb{R} \rightarrow[0,1]$ if

$\lim _{n \rightarrow \infty} m_{(n)}\left(x_{n}((-\infty, t))\right)=F(t)$

for each $t \in \mathbb{R}$. If $\left\{x_{n}: \mathcal{B}(\mathbb{R}) \rightarrow M_{(n)}\right\}_{n \in \mathbb{N}}$ is convergent in distribution to the cumulative distribution function of the standard normal distribution $\Phi$, then we write $x_{n} \rightarrow N(0,1)$ in distribution, as $n \rightarrow \infty$.

We recall generalized versions of MV-algebraic central limit theorems and the Feller theorem proved in Nowak and Hryniewicz (2015).

Theorem 3 (Lindeberg CLT) Let us assume that a TA $\left\{x_{n j}\right\}_{n \in \mathbb{N}, j \in \mathbb{N}\left[k_{n}\right]}$ satisfies (3) and the Lindeberg condition (4). Then

$\frac{1}{s_{n}}\left(\sum_{j=1}^{k_{n}} x_{n j}-\sum_{j=1}^{k_{n}} \mathbb{E}_{(n)}\left(x_{n j}\right)\right) \rightarrow N(0,1)$

in distribution, as $n \rightarrow \infty$.

Theorem 4 (Lyapunov CLT) Let us assume that a TA $\left\{x_{n j}\right\}_{n \in \mathbb{N}, j \in \mathbb{N}\left[k_{n}\right]}$ satisfies (3) and Lyapunov's condition (5). Then

$\frac{1}{s_{n}}\left(\sum_{j=1}^{k_{n}} x_{n j}-\sum_{j=1}^{k_{n}} \mathbb{E}_{(n)}\left(x_{n j}\right)\right) \rightarrow N(0,1)$

in distribution, as $n \rightarrow \infty$.

Theorem 5 (Feller) Let $\left\{x_{n j}\right\}_{n \in \mathbb{N}, j \in \mathbb{N}\left[k_{n}\right]}$ be a TA satisfying (3) and such that for each $\varepsilon>0$

$\max _{1 \leq j \leq k_{n}}\left(m_{(n)}\right)_{x_{n j}}\left(\left(-\infty,-\varepsilon s_{n}\right) \cup\left(\varepsilon s_{n}, \infty\right)\right) \rightarrow 0$,

as $n \rightarrow \infty$. If

$\frac{1}{s_{n}}\left(\sum_{j=1}^{k_{n}} x_{n j}-\sum_{j=1}^{k_{n}} \mathbb{E}_{(n)}\left(x_{n j}\right)\right) \rightarrow N(0,1)$

in distribution, as $n \rightarrow \infty$, then the Lindeberg condition (4) holds.

\section{IV-probability}

\subsection{Basic definitions and theorems}

Definition 12 Let $(\Omega, \mathcal{S})$ be a measurable space. By an interval-valued event (for short $I V$-event) we mean any pair $A=\left(\mu_{A}, v_{A}\right)$ of $\mathcal{S}$-measurable, [0,1]-valued functions such that $\mu_{A} \leq v_{A}$. We denote by $\mathcal{V}(\Omega, \mathcal{S})$ the set of all IV-events and we introduce the following operations on $\mathcal{V}(\Omega, \mathcal{S})$. For $A=\left(\mu_{A}, v_{A}\right), B=\left(\mu_{B}, v_{B}\right) \in \mathcal{V}(\Omega, \mathcal{S})$, $\left\{A_{n}\right\}_{n \in \mathbb{N}}=\left\{\left(\mu_{A_{n}}, v_{A_{n}}\right)\right\}_{n \in \mathbb{N}} \subset \mathcal{V}(\Omega, \mathcal{S})$ :

$A \oplus B=\left(\mu_{A} \oplus \mu_{B}, v_{A} \oplus v_{B}\right)$

$$
=\left(\left(\mu_{A}+\mu_{B}\right) \wedge 1,\left(v_{A}+v_{B}\right) \wedge 1\right) \text {; }
$$

$A \odot B=\left(\mu_{A} \odot \mu_{B}, v_{A} \odot v_{B}\right)$

$$
=\left(\left(\mu_{A}+\mu_{B}-1\right) \vee 0,\left(v_{A}+v_{B}-1\right) \vee 0\right)
$$

and we write $A_{n} \nearrow A \Leftrightarrow \mu_{A_{n}} \nearrow \mu_{A}, v_{A_{n}} \nearrow v_{A}$. Moreover, we consider the product

$A \cdot B=\left(\mu_{A} \mu_{B}, v_{A} v_{B}\right)$.

$\mathcal{V}(\Omega, \mathcal{S})$ is ordered as follows:

$A \leq B \Leftrightarrow \mu_{A} \leq \mu_{B}, \quad v_{A} \leq v_{B}$

The following theorem from Kuková (2011) states that $\mathcal{V}(\Omega, \mathcal{S})$ can be embedded to an MV-algebra.

\section{Theorem 6 Let}

$\mathcal{G}=\left\{A=\left(\mu_{A}, v_{A}\right) ; \mu_{A}, v_{A}: \Omega \rightarrow \mathbb{R}\right\}$

and the summation be defined by the formula

$A+B=\left(\mu_{A}+\mu_{B}, v_{A}+v_{B}\right) \quad$ for $A, B \in \mathcal{G}$.

Let the partial order on $\mathcal{G}$ be defined by

$A \leq B \Leftrightarrow \mu_{A} \leq \mu_{B} \wedge v_{A} \leq v_{B} \quad$ for $A, B \in \mathcal{G}$.

Let - denote the inverse operation to,$+ \mathbf{0}_{\Omega}=\left(0_{\Omega}, 0_{\Omega}\right)$ be the neutral element of + , and $\mathbf{1}_{\Omega}=\left(1_{\Omega}, 1_{\Omega}\right)$. Let $\mathcal{M}(\Omega, \mathcal{S})$ be an interval in $\mathcal{G}, \mathcal{M}(\Omega, \mathcal{S})=\left[\mathbf{0}_{\mathbf{\Omega}}, \mathbf{1}_{\mathbf{\Omega}}\right]$, with the operations

$A \oplus B=\left(\left(\mu_{A}+\mu_{B}\right) \wedge 1,\left(v_{A}+v_{B}\right) \wedge 1\right) ;$

$A \odot B=\left(\left(\mu_{A}+\mu_{B}-1\right) \vee 0,\left(v_{A}+v_{B}-1\right) \vee 0\right)$.

Then the system

$\left(\mathcal{M}(\Omega, \mathcal{S}), \oplus, \odot, \leq, \mathbf{0}_{\mathbf{\Omega}}, \mathbf{1}_{\mathbf{\Omega}}\right)$

is an $M V$-algebra and $\mathcal{V}(\Omega, \mathcal{S}) \subset \mathcal{M}(\Omega, \mathcal{S})$. 
We recall the notions of state, probability and observable for IV-events from Kuková (2011), Samuelčík and Hollá (2013), which we call IV-state, IV-probability and IVobservable, respectively, in this paper.

Definition 13 An IV-state on $\mathcal{V}(\Omega, \mathcal{S})$ is a map $m$ : $\mathcal{V}(\Omega, \mathcal{S}) \rightarrow[0,1]$ satisfying the following conditions for all $A, B \in \mathcal{V}(\Omega, \mathcal{S})$ and $\left\{A_{n}\right\}_{n=1}^{\infty} \subset \mathcal{V}(\Omega, \mathcal{S})$ :

(1) $m\left(\mathbf{1}_{\boldsymbol{\Omega}}\right)=1, m\left(\mathbf{0}_{\boldsymbol{\Omega}}\right)=0$;

(2) $A \odot B=\mathbf{0}_{\mathbf{\Omega}} \Rightarrow m(A \oplus B)=m(A)+m(B)$;

(3) if $A_{n} \nearrow A$, then $m\left(A_{n}\right) \nearrow m(A)$.

Let $\mathcal{J}$ be the family of all closed subintervals of $[0,1]$.

Definition 14 An $I V$-probability is a mapping $\mathfrak{P}: \mathcal{V}(\Omega, \mathcal{S})$ $\rightarrow \mathcal{J}$ satisfying the following conditions for all $A, B \in$ $\mathcal{V}(\Omega, \mathcal{S})$ and $\left\{A_{n}\right\}_{n=1}^{\infty} \subset \mathcal{V}(\Omega, \mathcal{S})$ :

(1) $\mathfrak{P}\left(\mathbf{1}_{\boldsymbol{\Omega}}\right)=[1,1], \mathfrak{P}\left(\mathbf{0}_{\boldsymbol{\Omega}}\right)=[0,0]$;

(2) $A \odot B=\mathbf{0}_{\boldsymbol{\Omega}} \Rightarrow \mathfrak{P}(A \oplus B)=\mathfrak{P}(A)+\mathfrak{P}(B)$;

(3) if $A_{n} \nearrow A$, then $\mathfrak{P}\left(A_{n}\right) \nearrow \mathfrak{P}(A)$.

An IV-probability space is a pair $(\mathcal{V}(\Omega, \mathcal{S}), \mathfrak{P})$, where $\mathfrak{P}$ is an $I V$-probability on $\mathcal{V}(\Omega, \mathcal{S})$. We will use the notation $\mathfrak{P}(A)=\left[\mathfrak{P}^{b}(A), \mathfrak{P}^{\natural}(A)\right]$ for each $A \in \mathcal{V}(\Omega, \mathcal{S})$.

It is easy to verify that if $\mathfrak{P}: \mathcal{V}(\Omega, \mathcal{S}) \rightarrow \mathcal{J}$ is an IVprobability, then the mappings $\mathfrak{P}^{b}$ and $\mathfrak{P}^{\natural}$ are IV-states on $\mathcal{V}(\Omega, \mathcal{S})$

In Sect. 4, we will use the following lemma.

Lemma 1 Let $\hat{P}$ be a probability measure defined on a measurable space $(\Omega, \mathcal{S})$. Then $\mathfrak{P}_{\hat{p}}: \mathcal{V}(\Omega, \mathcal{S}) \rightarrow \mathcal{J}$ of the form:

$\mathfrak{P}_{\hat{P}}((\mu, \nu))=\left[\int_{\Omega} \mu \mathrm{d} \hat{P}, \int_{\Omega} \nu \mathrm{d} \hat{P}\right], \quad(\mu, \nu) \in \mathcal{V}(\Omega, \mathcal{S})$,

is an IV-probability.

Proof It is obvious that $\mathfrak{P}_{\hat{P}}$ satisfies condition (1). Let $A \odot$ $B=\mathbf{0}_{\Omega}$ for $A, B \in \mathcal{V}(\Omega, \mathcal{S})$. Then for arbitrary $\omega \in \Omega$

$\mu_{A}(\omega)+\mu_{B}(\omega) \leq 1$ and $v_{A}(\omega)+v_{B}(\omega) \leq 1$.

Therefore,

$$
\begin{aligned}
\mathfrak{P}_{\hat{P}}(A \oplus B) & =\left[\int_{\Omega}\left[\left(\mu_{A}+\mu_{B}\right) \wedge 1\right] \mathrm{d} \hat{P}, \int_{\Omega}\left[\left(v_{A}+v_{B}\right) \wedge 1\right] \mathrm{d} \hat{P}\right] \\
= & {\left[\int_{\Omega}\left(\mu_{A}+\mu_{B}\right) \mathrm{d} \hat{P}, \int_{\Omega}\left(v_{A}+v_{B}\right) \mathrm{d} \hat{P}\right] }
\end{aligned}
$$

$$
\begin{aligned}
& =\left[\int_{\Omega} \mu_{A} \mathrm{~d} \hat{P}, \int_{\Omega} v_{A} \mathrm{~d} \hat{P}\right]+\left[\int_{\Omega} \mu_{B} \mathrm{~d} \hat{P}, \int_{\Omega} v_{B} \mathrm{~d} \hat{P}\right] \\
& =\mathfrak{P}_{\hat{P}}(A)+\mathfrak{P}_{\hat{P}}(B) .
\end{aligned}
$$

Thus, condition (2) is satisfied.

Let $A \in \mathcal{V}(\Omega, \mathcal{S}),\left\{A_{n}\right\}_{n=1}^{\infty} \subset \mathcal{V}(\Omega, \mathcal{S})$ and $A_{n} \nearrow A$. Then

$$
\begin{aligned}
\mathfrak{P}_{\hat{P}}\left(A_{n}\right)= & {\left[\int_{\Omega} \mu_{A_{n}} \mathrm{~d} \hat{P}, \int_{\Omega} v_{A_{n}} \mathrm{~d} \hat{P}\right] } \\
\nearrow & \nearrow\left[\int_{\Omega} \mu_{A} \mathrm{~d} \hat{P}, \int_{\Omega} v_{A} \mathrm{~d} \hat{P}\right]=\mathfrak{P}(A)
\end{aligned}
$$

by the Dominated Convergence Theorem. Therefore, condition (3) is also fulfilled.

The IV-probability $\mathfrak{P}_{\hat{P}}((\mu, v))$ is a modification of the probability of IF-events, which was considered by Szmidt and Kacprzyk (1999a,b), Grzegorzewski and Mrówka (2002) and generalized by Nowak (2003, 2004a, b).

Definition 15 An IV-observable is a mapping $x: \mathcal{B}(\mathbb{R}) \rightarrow$ $\mathcal{V}(\Omega, \mathcal{S})$ satisfying the following conditions:

(1) $x(\mathbb{R})=\mathbf{1}_{\boldsymbol{\Omega}}, x(\emptyset)=\mathbf{0}_{\boldsymbol{\Omega}} ;$

(2) whenever $A, B \in \mathcal{B}(\mathbb{R})$ and $A \cap B=\emptyset$, then

$$
x(A) \odot x(B)=\mathbf{0}_{\mathbf{\Omega}} \text { and } \quad x(A \cup B)=x(A) \oplus x(B) ;
$$

(3) for all $A, A_{1}, A_{2}, \ldots \in \mathcal{B}(\mathbb{R})$

if $A_{n} \nearrow A$, then $x\left(A_{n}\right) \nearrow x(A)$.

Definition 16 If $x_{1}, x_{2}, \ldots, x_{n}: \mathcal{B}(\mathbb{R}) \rightarrow \mathcal{V}(\Omega, \mathcal{S})$ are IVobservables, then their joint $I V$-observable is the map $h$ : $\mathcal{B}\left(\mathbb{R}^{n}\right) \rightarrow \mathcal{V}(\Omega, \mathcal{S})$ satisfying the following conditions:

(1) $h\left(\mathbb{R}^{n}\right)=\mathbf{1}_{\mathbf{\Omega}}, h(\emptyset)=\mathbf{0}_{\boldsymbol{\Omega}}$;

(2) whenever $A, B \in \mathcal{B}\left(\mathbb{R}^{n}\right)$ and $A \cap B=\emptyset$, then

$$
h(A) \odot h(B)=\mathbf{0}_{\boldsymbol{\Omega}}, h(A \cup B)=h(A) \oplus h(B) ;
$$

(3) for all $A, A_{1}, A_{2}, \ldots \in \mathcal{B}\left(\mathbb{R}^{n}\right)$

if $A_{n} \nearrow A$, then $h\left(A_{n}\right) \nearrow h(A)$;

(4) for all $C_{1}, C_{2}, \ldots, C_{n} \in \mathcal{B}(\mathbb{R})$

$$
\begin{aligned}
& h\left(C_{1} \times C_{2} \times \cdots \times C_{n}\right) \\
& \quad=x_{1}\left(C_{1}\right) \cdot x_{2}\left(C_{2}\right) \cdot \ldots \cdot x_{n}\left(C_{n}\right) .
\end{aligned}
$$

The following theorems and proposition from Kuková (2011) (see Theorem 2) and Samuelčík and Hollá (2013) (see Proposition 1 and Theorem 1), concern properties of the notions defined above. 
Theorem 7 Let $\bar{p}: \mathcal{M}(\Omega, \mathcal{S}) \rightarrow[0,1]$ be defined by the formula

$\bar{p}(A)=\bar{p}\left(\mu_{A}, v_{A}\right)=p\left(\mu_{A}, 1\right)-p\left(0,1-v_{A}\right)$,

where $p: \mathcal{V}(\Omega, \mathcal{S}) \rightarrow[0,1]$ is an IV-state on $\mathcal{V}(\Omega, \mathcal{S})$. Then

1. for arbitrary $A \in \mathcal{V}(\Omega, \mathcal{S}) \bar{p}(A)=p(A)$,

2. $\bar{p}$ is a state on $\mathcal{M}(\Omega, \mathcal{S})$.

Proposition 1 If $x: \mathcal{B}(\mathbb{R}) \rightarrow \mathcal{V}(\Omega, \mathcal{S})$ is an IV-observable and $p: \mathcal{V}(\Omega, \mathcal{S}) \rightarrow[0,1]$ is an IV-state, then the mapping $p_{x}=p \circ x: \mathcal{B}(\mathbb{R}) \rightarrow[0,1]$ defined by the formula

$p_{x}(A)=p(x(A))$

is a probability measure.

Theorem 8 For any IV-observables

$x_{1}, x_{2}, \ldots, x_{n}: \mathcal{B}(\mathbb{R}) \rightarrow \mathcal{V}(\Omega, \mathcal{S})$

there exists their joint IV-observable

$h: \mathcal{B}\left(\mathbb{R}^{n}\right) \rightarrow \mathcal{V}(\Omega, \mathcal{S})$

The following remarks will be very useful in the further part of the paper.

Remark 2 Since $\mathcal{V}(\Omega, \mathcal{S}) \subset \mathcal{M}(\Omega, \mathcal{S})$, any IV-observable $x: \mathcal{B}(\mathbb{R}) \rightarrow \mathcal{V}(\Omega, \mathcal{S})$ is an observable in the sense of the MV-algebraic probability theory. Furthermore,

$\mathfrak{P}_{x}^{b}=\mathfrak{P}^{b} \circ x, \mathfrak{P}_{x}^{\natural}=\mathfrak{P}^{\natural} \circ x: \mathcal{B}(\mathbb{R}) \rightarrow[0,1]$

are probability measures.

Remark 3 Let $x_{1}, x_{2}, \ldots, x_{n}: \mathcal{B}(\mathbb{R}) \rightarrow \mathcal{V}(\Omega, \mathcal{S})$ be IVobservables. Let $g: \mathbb{R}^{n} \rightarrow \mathbb{R}$ be a Borel measurable function and let $h: \mathcal{B}\left(\mathbb{R}^{n}\right) \rightarrow \mathcal{V}(\Omega, \mathcal{S})$ be the joint observable of $x_{1}, x_{2}, \ldots, x_{n}$. Then

$g\left(x_{1}, x_{2}, \ldots, x_{n}\right)=h \circ g^{-1}$

is an IV-observable.

Definition 17 IV-observables

$x_{1}, x_{2}, \ldots, x_{n}: \mathcal{B}(\mathbb{R}) \rightarrow \mathcal{V}(\Omega, \mathcal{S})$

are independent (with respect to $\mathfrak{P}$ ) if there exists an $n$ dimensional IV-observable $h: \mathcal{B}\left(\mathbb{R}^{n}\right) \rightarrow \mathcal{V}(\Omega, \mathcal{S})$ such that for all $C_{1}, C_{2}, \ldots, C_{n} \in \mathcal{B}(\mathbb{R})$

$$
\begin{aligned}
\mathfrak{P}^{\mathrm{b}}\left(h\left(C_{1} \times C_{2} \times \cdots \times C_{n}\right)\right) & =\mathfrak{P}_{x_{1}}^{\mathrm{b}}\left(C_{1}\right) \cdot \mathfrak{P}_{x_{2}}^{\mathrm{b}}\left(C_{2}\right) \\
& \cdots \cdot \mathfrak{P}_{x_{n}}^{\mathrm{b}}\left(C_{n}\right), \\
\mathfrak{P}^{\natural}\left(h\left(C_{1} \times C_{2} \times \cdots \times C_{n}\right)\right) & =\mathfrak{P}_{x_{1}}^{\natural}\left(C_{1}\right) \cdot \mathfrak{P}_{x_{2}}^{\natural}\left(C_{2}\right) \\
& \ldots \cdot \mathfrak{P}_{x_{n}}^{\natural}\left(C_{n}\right) .
\end{aligned}
$$

Definition 18 Let $\mathfrak{P}: \mathcal{V}(\Omega, \mathcal{S}) \rightarrow \mathcal{J}$ be an IV-probability and $x: \mathcal{B}(\mathbb{R}) \rightarrow \mathcal{V}(\Omega, \mathcal{S})$ be an IV-observable. Then $x$ is said to be integrable if the expectations

$\mathbb{E}^{b}(x)=\int_{\mathbb{R}} t \mathfrak{P}_{x}^{b}(\mathrm{~d} t), \quad \mathbb{E}^{\natural}(x)=\int_{\mathbb{R}} t \mathfrak{P}_{x}^{\natural}(\mathrm{d} t)$

exist. We say that $x$ is square-integrable if $\int_{\mathbb{R}} t^{2} \mathfrak{P}_{x}^{b}(\mathrm{~d} t)$ and $\int_{\mathbb{R}} t^{2} \mathfrak{P}_{x}^{\natural}(\mathrm{d} t)$ exist. If $x$ is square-integrable, then the variances of $x$ also exist and are described by the equalities

$$
\begin{aligned}
\mathbb{D}^{b, 2}(x) & =\int_{\mathbb{R}} t^{2} \mathfrak{P}_{x}^{b}(\mathrm{~d} t)-\left(\mathbb{E}^{\mathrm{b}}(x)\right)^{2} \\
& =\int_{\mathbb{R}}\left(t-\mathbb{E}^{b}(x)\right)^{2} \mathfrak{P}_{x}^{b}(\mathrm{~d} t), \\
\mathbb{D}^{\natural, 2}(x) & =\int_{\mathbb{R}} t^{2} \mathfrak{P}_{x}^{\natural}(\mathrm{d} t)-\left(\mathbb{E}^{\natural}(x)\right)^{2} \\
& =\int_{\mathbb{R}}\left(t-\mathbb{E}^{\natural}(x)\right)^{2} \mathfrak{P}_{x}^{\natural}(\mathrm{d} t) .
\end{aligned}
$$

We write $x \in L_{\mathfrak{P}}^{p_{1}, p_{2}}$ for $p_{1}, p_{2} \geq 1$ if $\int_{\mathbb{R}}|t|^{p_{1}} \mathfrak{P}_{x}^{b}(\mathrm{~d} t)<\infty$ and $\int_{\mathbb{R}}|t|^{p_{2}} \mathfrak{P}_{x}^{\natural}(\mathrm{d} t)<\infty$. Finally, we use the notation $x \in$ $L_{\mathfrak{P}}^{p}$ instead of $x \in L_{\mathfrak{P}}^{p, p}$ for $p \geq 1$.

The following lemma concerns the form of the expected value of a Borel function of an IV-observable.

Lemma 2 Let $\mathfrak{P}: \mathcal{V}(\Omega, \mathcal{S}) \rightarrow \mathcal{J}$ be an IV-probability, $\varphi$ be an $\mathbb{R}$-valued Borel function, which domain is the whole set of real numbers $\mathbb{R}, x: \mathcal{B}(\mathbb{R}) \rightarrow \mathcal{V}(\Omega, \mathcal{S})$ be an IV-observable and $y=\varphi(x)=x \circ \varphi^{-1}$. Then $\mathbb{E}^{b}(y)$ exists if and only if $\int_{\mathbb{R}}|\varphi(t)| \mathfrak{P}_{x}^{\mathrm{b}}(\mathrm{d} t)<\infty$ and then $\mathbb{E}^{\mathrm{b}}(y)=\int_{\mathbb{R}} \varphi(t) \mathfrak{P}_{x}^{\mathrm{b}}(\mathrm{d} t)$. Moreover, the analogous assertion holds for $\mathbb{E}^{\natural}(y)$ and the corresponding probability measure $\mathfrak{P}_{x}^{\natural}$.

Proof We use Theorem 1 for

$(X, \mathcal{X})=\left(X^{\prime}, \mathcal{X}^{\prime}\right)=(\mathbb{R}, \mathcal{B}(\mathbb{R})), \quad T=\varphi \quad$ and $\quad f(t)=t$.

Then $\mu=\mathfrak{P}_{x}^{b}$ is a probability measure. Moreover, by straightforward computations one can verify the equality $\mu T^{-1}=\mathfrak{P}_{\varphi(x)}^{b}=\mathfrak{P}_{y}^{b}$, which ends the proof. One can prove analogously the assertion for $\mathbb{E}^{\natural}(y)$.

\subsection{Central limit theorems}

We denote by $\left\{k_{n}\right\}_{n \in \mathbb{N}}$ a fixed sequence of positive integers and assume that $\lim _{n \rightarrow \infty} k_{n}=\infty$. 
Let $\left\{\left(\mathcal{V}\left(\Omega_{(n)}, \mathcal{S}_{(n)}\right), \mathfrak{P}_{(n)}\right)\right\}_{n \in \mathbb{N}}$ be a sequence of IVprobability spaces. For each $n \in \mathbb{N}$ and an observable $x: \mathcal{B}(\mathbb{R}) \rightarrow \mathcal{V}\left(\Omega_{(n)}, \mathcal{S}_{(n)}\right)$, we use the symbols $\mathbb{E}_{(n)}^{\mathrm{b}}(x)$, $\mathbb{E}_{(n)}^{\natural}(x)$ to denote the expected values of $x$ and the symbols $\mathbb{D}_{(n)}^{2, b}(x), \mathbb{D}_{(n)}^{2, \natural}(x)$ to denote the variances of $x$ with respect to $\mathfrak{P}_{(n)}^{\mathrm{b}}$ and $\mathfrak{P}_{(n)}^{\natural}$, respectively.

For $n \in \mathbb{N}$, constants $\varepsilon, s>0$, and an arbitrary IVobservable $x: \mathcal{B}(\mathbb{R}) \rightarrow \mathcal{V}\left(\Omega_{(n)}, \mathcal{S}_{(n)}\right)$ belonging to $L_{\mathfrak{P}_{(n)}}^{2}$ we consider the following $\mathbb{R}$-valued functions:

$$
\begin{aligned}
& \mathfrak{l}_{n, b}^{x}(\varepsilon, s)=\mathbb{E}_{(n)}^{\mathrm{b}}\left(\left(x-\mathbb{E}_{(n)}^{\mathrm{b}}(x)\right)^{2} I_{\left|x-\mathbb{E}_{(n)}^{\mathrm{b}}(x)\right|>\varepsilon s}\right), \\
& \mathfrak{l}_{n, \sharp}^{x}(\varepsilon, s)=\mathbb{E}_{(n)}^{\natural}\left(\left(x-\mathbb{E}_{(n)}^{\natural}(x)\right)^{2} I_{\left|x-\mathbb{E}_{(n)}^{\natural}(x)\right|>\varepsilon s}\right) .
\end{aligned}
$$

Lemma 2 implies that $\mathfrak{l}_{n, b}^{x}$ and $\mathfrak{l}_{n, \sharp}^{x}$ are well-defined.

Definition 19 Let for each $n \in \mathbb{N}\left\{x_{n j}\right\}_{j \in \mathbb{N}\left[k_{n}\right]}$ be a sequence of independent (with respect to $\mathfrak{P}_{(n)}$ ) IV-observables of $\mathcal{V}\left(\Omega_{(n)}, \mathcal{S}_{(n)}\right)$. Then $\left\{x_{n j}\right\}_{n \in \mathbb{N}, j \in \mathbb{N}\left[k_{n}\right]}$ is called a triangular array of independent IV-observables (TVI for short).

Definition 20 Let $\left\{x_{n j}\right\}_{n \in \mathbb{N}, j \in \mathbb{N}\left[k_{n}\right]}$ be a TVI satisfying the following conditions for each $n \in \mathbb{N}$

$$
\begin{aligned}
& x_{n j} \in L_{\mathfrak{P}_{(n)}}^{2}, \quad j \in \mathbb{N}\left[k_{n}\right], \\
& s_{n}^{2, b}=\sum_{j=1}^{k_{n}} \mathbb{D}_{(n)}^{2, b}\left(x_{n j}\right), \quad s_{n}^{2, \natural}=\sum_{j=1}^{k_{n}} \mathbb{D}_{(n)}^{2, \natural}\left(x_{n j}\right) \in(0, \infty) .
\end{aligned}
$$

Then $\left\{x_{n j}\right\}_{n \in \mathbb{N}, j \in \mathbb{N}\left[k_{n}\right]}$ is said to satisfy the Lindeberg condition if for each $\varepsilon>0$

$\mathfrak{L}_{n}(\varepsilon)=\mathfrak{L}_{n}^{b}(\varepsilon)+\mathfrak{L}_{n}^{\natural}(\varepsilon) \rightarrow 0, \quad$ as $n \rightarrow \infty$,

where

$$
\begin{aligned}
& \mathfrak{L}_{n}^{b}(\varepsilon)=\frac{1}{s_{n}^{2, b}} \sum_{j=1}^{k_{n}} \mathrm{r}_{n, b}^{x_{n j}}\left(\varepsilon, s_{n}^{b}\right), \\
& \mathfrak{L}_{n}^{\natural}(\varepsilon)=\frac{1}{s_{n}^{2, \natural}} \sum_{j=1}^{k_{n}} \mathrm{r}_{n, \sharp}^{x_{n j}}\left(\varepsilon, s_{n}^{\natural}\right), \\
& s_{n}^{b}=\sqrt{s_{n}^{2, b}} \text { and } s_{n}^{\natural}=\sqrt{s_{n}^{2,,}} .
\end{aligned}
$$

Definition 21 Let $\left\{x_{n j}\right\}_{n \in \mathbb{N}, j \in \mathbb{N}\left[k_{n}\right]}$ be a TVI fulfilling (7)(8). Then the array $\left\{x_{n j}\right\}_{n \in \mathbb{N}, j \in \mathbb{N}\left[k_{n}\right]}$ is said to satisfy the Lyapunov condition if there exist positive constants $\delta_{1}$ and $\delta_{2}$ such that

$\frac{1}{s_{n}^{2+\delta_{1}, \mathrm{~b}}} \sum_{j=1}^{k_{n}} \mathbb{E}_{(n)}^{\mathrm{b}}\left(\left|x_{n j}-\mathbb{E}_{(n)}^{\mathrm{b}}\left(x_{n j}\right)\right|^{2+\delta_{1}}\right)$

$$
+\frac{1}{s_{n}^{2+\delta_{2}, \natural}} \sum_{j=1}^{k_{n}} \mathbb{E}_{(n)}^{\natural}\left(\left|x_{n j}-\mathbb{E}_{(n)}^{\natural}\left(x_{n j}\right)\right|^{2+\delta_{2}}\right) \rightarrow 0,
$$

as $n \rightarrow \infty$,

where $s_{n}^{2+\delta_{1}, \mathrm{~b}}=\left(s_{n}^{b}\right)^{2+\delta_{1}}$ and $s_{n}^{2+\delta_{2}, \natural}=\left(s_{n}^{\natural}\right)^{2+\delta_{2}}$.

The next two theorems are IV-probabilistic versions of central limit theorems.

Theorem 9 (Lindeberg CLT) Let $\left\{x_{n j}\right\}_{n \in \mathbb{N}, j \in \mathbb{N}\left[k_{n}\right]}$ be a TVI satisfying (7)-(8) as well as the Lindeberg condition (9). Then for $t \in \mathbb{R}$

$\mathfrak{P}_{(n)}^{\mathrm{b}}\left(\frac{\sum_{j=1}^{k_{n}} x_{n j}-\sum_{j=1}^{k_{n}} \mathbb{E}_{(n)}^{\mathrm{b}}\left(x_{n j}\right)}{s_{n}^{\mathrm{b}}}((-\infty, t))\right)$
$\quad \rightarrow \Phi(t)$, as $n \rightarrow \infty$

$\mathfrak{P}_{(n)}^{\natural}\left(\frac{\sum_{j=1}^{k_{n}} x_{n j}-\sum_{j=1}^{k_{n}} \mathbb{E}_{(n)}^{\natural}\left(x_{n j}\right)}{s_{n}^{\natural}}((-\infty, t))\right)$

$\rightarrow \Phi(t)$, as $n \rightarrow \infty$.

Proof We consider the sequence of MV-algebras $\mathcal{M}\left(\Omega_{(n)}, \mathcal{S}_{(n)}\right)$ with states $\overline{\mathfrak{P}}_{(n)}^{\mathrm{b}}, n \in \mathbb{N}$. From Theorem 7, it follows that for arbitrary $n \in \mathbb{N}$ one can find a state $\overline{\mathfrak{P}}_{(n)}^{b}$ : $\mathcal{M}\left(\Omega_{(n)}, \mathcal{S}_{(n)}\right) \rightarrow[0,1]$ such that $\overline{\mathfrak{P}}_{(n)}^{\mathrm{b}} \mid \mathcal{V}\left(\Omega_{(n)}, \mathcal{S}_{(n)}\right)=\mathfrak{P}_{(n)}^{\mathrm{b}}$. Since

$\mathcal{V}\left(\Omega_{(n)}, \mathcal{S}_{(n)}\right) \subset \mathcal{M}\left(\Omega_{(n)}, \mathcal{S}_{(n)}\right)$,

the array $\left\{x_{n j}\right\}_{n \in \mathbb{N}, j \in \mathbb{N}\left[k_{n}\right]}$ is a TA of MV-algebras

$\left\{\mathcal{M}\left(\Omega_{(n)}, \mathcal{S}_{(n)}\right)\right\}_{n \in \mathbb{N}}$. For arbitrary $n \in \mathbb{N}, j \in \mathbb{N}\left[k_{n}\right]$ $\left(\overline{\mathfrak{P}}_{(n)}^{\mathrm{b}}\right)_{x_{n j}}$ and $\left(\mathfrak{P}_{(n)}^{\mathrm{b}}\right)_{x_{n j}}$ coincide. Consequently, for arbitrary $n \in \mathbb{N}$ expected values $\mathbb{E}_{(n)}^{b}\left(x_{n j}\right), j \in \mathbb{N}\left[k_{n}\right]$, variances $\mathbb{D}_{(n)}^{2, b}\left(x_{n j}\right), j \in \mathbb{N}\left[k_{n}\right]$ as well as $\mathfrak{L}_{n}^{b}(\varepsilon), \mathfrak{L}_{n}^{\natural}(\varepsilon)$ coincide with their MV-algebraic counterparts for the state $\overline{\mathfrak{P}}_{(n)}^{b}$. Thus, the Lindeberg condition (9) implies its MV-algebraic counterpart

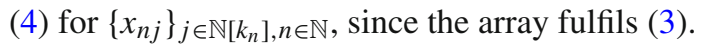

Let $\left\{Z_{n}^{\mathrm{b}}\right\}_{n \in \mathbb{N}}$ be the sequence of $\mathcal{V}\left(\Omega_{(n)}, \mathcal{S}_{(n)}\right)$-valued observables given by the formula:

$Z_{n}^{\mathrm{b}}=\frac{\sum_{j=1}^{k_{n}} x_{n j}-\sum_{j=1}^{k_{n}} \mathbb{E}_{(n)}^{\mathrm{b}}\left(x_{n j}\right)}{s_{n}^{\mathrm{b}}}, n \in \mathbb{N}$.

Clearly, for each $n \in \mathbb{N}$ and $t \in \mathbb{R}$

$\overline{\mathfrak{P}}_{(n)}^{\mathrm{b}}\left(Z_{n}^{\mathrm{b}}((-\infty, t))\right)=\mathfrak{P}_{(n)}^{\mathrm{b}}\left(Z_{n}^{\mathrm{b}}((-\infty, t))\right)$.

By Theorem 3,

$\overline{\mathfrak{P}}_{(n)}^{\mathrm{b}}\left(Z_{n}^{\mathrm{b}}((-\infty, t))\right) \rightarrow \Phi(t)$, as $n \rightarrow \infty$ 
for $t \in \mathbb{R}$ and therefore the convergence (11) holds. The convergence (12) we obtain analogously.

Theorem 10 (Lyapunov CLT) Let $\left\{x_{n j}\right\}_{n \in \mathbb{N}, j \in \mathbb{N}\left[k_{n}\right]}$ be a TVI satisfying (7)-(8) and Lyapunov's condition (10). Then for each $t \in \mathbb{R}$ (11)-(12) hold.

Proof As we noticed previously, $\left\{x_{n j}\right\}_{n \in \mathbb{N}, j \in \mathbb{N}\left[k_{n}\right]}$ is a TA of the MV-algebras $\left\{\mathcal{M}\left(\Omega_{(n)}, \mathcal{S}_{(n)}\right)\right\}_{n \in \mathbb{N}}$ with states $\overline{\mathfrak{P}}_{(n)}^{b}, n=$ $1,2, \ldots$ For any positive integer $n$ expected values $\mathbb{E}_{(n)}^{b}\left(x_{n j}\right)$, $j \in \mathbb{N}\left[k_{n}\right]$, and variances $\mathbb{D}_{(n)}^{2, b}\left(x_{n j}\right), j \in \mathbb{N}\left[k_{n}\right]$, coincide with their MV-algebraic counterparts for the state $\overline{\mathfrak{P}}_{(n)}^{b}$. Thus, the TA $\left\{x_{n j}\right\}_{n \in \mathbb{N}, j \in \mathbb{N}\left[k_{n}\right]}$ of MV-algebras $\mathcal{M}\left(\Omega_{(n)}, \mathcal{S}_{(n)}\right)$ fulfils (3) and the Lyapunov condition (10) implies its counterpart (5) for this array, considered with states $\overline{\mathfrak{P}}_{(n)}^{b}$. Therefore, Theorem 4 implies

$\overline{\mathfrak{P}}_{(n)}^{b}\left(Z_{n}^{b}((-\infty, t))\right) \rightarrow \Phi(t)$, as $n \rightarrow \infty$

for arbitrary $t \in \mathbb{R}$, where $\left\{Z_{n}^{b}\right\}_{n \in \mathbb{N}}$ is the sequence of $\mathcal{V}\left(\Omega_{(n)}, \mathcal{S}_{(n)}\right)$-valued observables given by formula (13). The same reasoning as in the last part of the previous proof justifies (11). Analogously, we obtain (12).

The following theorem is an IV-probabilistic version of the Feller theorem.

Theorem 11 (Feller) Let $\left\{x_{n j}\right\}_{n \in \mathbb{N}, j \in \mathbb{N}\left[k_{n}\right]}$ be a TVI satisfying (7)-(8) and such that for each $\varepsilon>0$

$\lim _{n \rightarrow \infty} \max _{1 \leq j \leq k_{n}}\left(\mathfrak{P}_{(n)}^{b}\right)_{x_{n j}}\left(E\left(\varepsilon s_{n}^{b}\right)\right)=0$,

$\lim _{n \rightarrow \infty} \max _{1 \leq j \leq k_{n}}\left(\mathfrak{P}_{(n)}^{\natural}\right)_{x_{n j}}\left(E\left(\varepsilon s_{n}^{\natural}\right)\right)=0$,

where

$E(a)=(-\infty,-a) \cup(a, \infty)$

for $a>0$. Let us additionally assume that for $t \in \mathbb{R}$ the convergences (11) and (12) hold. Then the Lindeberg condition (9) is fulfilled.

Proof Since for any $n \in \mathbb{N}, j \in \mathbb{N}\left[k_{n}\right]\left(\overline{\mathfrak{P}}_{(n)}^{b}\right)_{x_{n j}}$ and $\left(\mathfrak{P}_{(n)}^{b}\right)_{x_{n j}}$ coincide, the equalities

$\lim _{n \rightarrow \infty} \max _{1 \leq j \leq k_{n}}\left(\overline{\mathfrak{P}}_{(n)}^{b}\right)_{x_{n j}}\left(E\left(\varepsilon s_{n}^{b}\right)\right)=0$,

$\lim _{n \rightarrow \infty} \max _{1 \leq j \leq k_{n}}\left(\overline{\mathfrak{P}}_{(n)}^{\natural}\right)_{x_{n j}}\left(E\left(\varepsilon s_{n}^{\natural}\right)\right)=0$

follows from (14) and (15). The TA $\left\{x_{n j}\right\}_{n \in \mathbb{N}, j \in \mathbb{N}\left[k_{n}\right]}$ of MValgebras $\mathcal{M}\left(\Omega_{(n)}, \mathcal{S}_{(n)}\right)$, considered with states $\overline{\mathfrak{P}}_{(n)}^{b}$ and $\overline{\mathfrak{P}}_{(n)}^{\natural}$, satisfies (3) and therefore Theorem 5 implies that for each $\varepsilon>0 \mathfrak{L}_{n}^{b}(\varepsilon) \rightarrow 0, \mathfrak{L}_{n}^{\natural}(\varepsilon) \rightarrow 0$, as $n \rightarrow \infty$. Thus, $\mathfrak{L}_{n}(\varepsilon)=\mathfrak{L}_{n}^{b}(\varepsilon)+\mathfrak{L}_{n}^{\natural}(\varepsilon) \rightarrow 0$, as $n \rightarrow \infty$, which finishes the proof.

\section{Applications}

In this section, we present and analyse three examples of arrays and sequences of IV-observables with convergent scaled sums or row sums. The first example is preceded by the central limit theorem for independent, identically distributed IV-observables with proof. In the second and third example, the considered observables are not identically distributed and therefore Theorem 12 cannot be applied for them. To prove the convergence in distribution of the considered scaled row sums to standard normal distribution, we use Theorem 9 and Theorem 10.

\subsection{Convergence of independent IV-observables with the same distributions}

Let us consider a sequence of independent IV-observables with the same distribution.

Theorem 12 Let $(\mathcal{V}(\Omega, \mathcal{S}), \mathfrak{P})$ be an IV-probability space. Let us assume that $\left\{x_{j}: \mathcal{B}(\mathbb{R}) \rightarrow \mathcal{V}(\Omega, \mathcal{S})\right\}_{j \in \mathbb{N}}$ is a sequence of independent IV-observables with the same distribution and variances

$\left(\sigma^{b}\right)^{2}=\mathbb{D}^{2, b}(x), \quad\left(\sigma^{\natural}\right)^{2}=\mathbb{D}^{2, \natural}(x)$

with respect to $\mathfrak{P}^{b}$ and $\mathfrak{P}^{\natural}$, respectively, where $0<\sigma^{b}, \sigma^{\natural}<$ $\infty$. Let $e^{b}=\mathbb{E}^{b}\left(x_{1}\right)$ and $e^{\natural}=\mathbb{E}^{\natural}\left(x_{1}\right)$. Then for $t \in \mathbb{R}$

$\mathfrak{P}^{b}\left(\frac{\sum_{j=1}^{n} x_{j}-n e^{b}}{\sigma^{b} \sqrt{n}}((-\infty, t))\right) \rightarrow \Phi(t)$, as $n \rightarrow \infty$,

$\mathfrak{P}^{\natural}\left(\frac{\sum_{j=1}^{n} x_{j}-n e^{\natural}}{\sigma^{\natural} \sqrt{n}}((-\infty, t))\right) \rightarrow \Phi(t)$, as $n \rightarrow \infty$.

Proof Let $\left\{\left(\mathcal{V}\left(\Omega_{(n)}, \mathcal{S}_{(n)}\right), \mathfrak{P}_{(n)}\right)\right\}_{n \in \mathbb{N}}$ be the constant sequence of IV-probability spaces, where $\Omega_{(n)}=\Omega, \mathcal{S}_{(n)}=\mathcal{S}$ for arbitrary $n \in \mathbb{N}$. Let for each $n \in \mathbb{N} k_{n}=n$ and $x_{n j}=$ $x_{j}, j \in \mathbb{N}[n]$. Then $\left\{x_{n j}\right\}_{n \in \mathbb{N}, j \in \mathbb{N}\left[k_{n}\right]}$ is a TVI with respect to the aforementioned constant sequence of IV-probability spaces. Moreover, $\left\{x_{n j}\right\}_{n \in \mathbb{N}, j \in \mathbb{N}\left[k_{n}\right]}$ satisfies conditions (7) and (8), where 
$s_{n}^{2, b}=n\left(\sigma^{b}\right)^{2}, \quad s_{n}^{2, \natural}=n\left(\sigma^{\natural}\right)^{2}$.

Furthermore, for each $n \in \mathbb{N}$ and $j \in \mathbb{N}[n]$

$$
\begin{aligned}
& \mathfrak{l}_{n, b}^{x_{n j}}\left(\varepsilon, s_{n}^{b}\right)=\mathbb{E}^{b}\left(\left(x_{1}-e^{b}\right)^{2} I_{\left.\left|x_{1}-e^{b}\right|>\varepsilon \sigma^{b} \sqrt{n}\right),}\right. \\
& \mathfrak{r}_{n, \natural}^{x_{n j}}\left(\varepsilon, s_{n}^{\natural}\right)=\mathbb{E}^{\natural}\left(\left(x_{1}-e^{\natural}\right)^{2} I_{\left|x_{1}-e^{\natural}\right|>\varepsilon \sigma^{\natural} \sqrt{n}}\right) .
\end{aligned}
$$

Clearly,

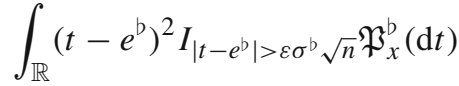

$$
\begin{aligned}
& \leq \int_{\mathbb{R}}\left(t-e^{b}\right)^{2} \mathfrak{P}_{x}^{b}(\mathrm{~d} t)=\left(\sigma^{b}\right)^{2}<\infty .
\end{aligned}
$$

Therefore,

$$
\begin{aligned}
\mathbb{E}^{b} & \left(\left(x_{1}-e^{b}\right)^{2} I_{\left|x_{1}-e^{b}\right|>\varepsilon \sigma^{b} \sqrt{n}}\right) \\
& =\int_{\mathbb{R}}\left(t-e^{b}\right)^{2} I_{\left|t-e^{b}\right|>\varepsilon \sigma^{b} \sqrt{n}} \mathfrak{P}_{x}^{b}(\mathrm{~d} t)
\end{aligned}
$$

by Lemma 2. Applying the Dominated Convergence Theorem, we obtain the convergence

$$
\begin{aligned}
\mathbb{E}^{b} & \left(\left(x_{1}-e^{b}\right)^{2} I_{\left|x_{1}-e^{b}\right|>\varepsilon \sigma^{b} \sqrt{n}}\right) \\
& =\int_{\mathbb{R}}\left(t-e^{b}\right)^{2} I_{\left|t-e^{b}\right|>\varepsilon \sigma^{b} \sqrt{n}} \mathfrak{P}_{x}^{b}(\mathrm{~d} t) \rightarrow 0, \text { as } n \rightarrow \infty .
\end{aligned}
$$

Thus,

$$
\begin{aligned}
\mathfrak{L}_{n}^{b}(\varepsilon) & =\frac{1}{s_{n}^{2, b}} \sum_{j=1}^{k_{n}} \mathfrak{r}_{n, b}^{x_{n j}}\left(\varepsilon, s_{n}^{b}\right) \\
& =\frac{1}{n\left(\sigma^{b}\right)^{2}} n \mathbb{E}^{b}\left(\left(x_{1}-e^{b}\right)^{2} I_{\left|x_{1}-e^{b}\right|>\varepsilon \sigma^{b} \sqrt{n}}\right) \\
& =\frac{1}{\left(\sigma^{b}\right)^{2}} \mathbb{E}^{b}\left(\left(x_{1}-e^{b}\right)^{2} I_{\left|x_{1}-e^{b}\right|>\varepsilon \sigma^{b} \sqrt{n}}\right) \rightarrow 0,
\end{aligned}
$$

as $n \rightarrow \infty$ and similarly

$$
\mathfrak{L}_{n}^{\natural}(\varepsilon)=\frac{1}{\left(\sigma^{\natural}\right)^{2}} \mathbb{E}^{b}\left(\left(x_{1}-e^{\natural}\right)^{2} I_{\left|x_{1}-e^{\natural}\right|>\varepsilon \sigma^{\natural} \sqrt{n}}\right) \rightarrow 0,
$$

as $n \rightarrow \infty$. Consequently, $\left\{x_{n j}\right\}_{n \in \mathbb{N}, j \in \mathbb{N}\left[k_{n}\right]}$ satisfies the Lindeberg condition (9). Therefore, Theorem 9 implies the convergences (18) and (19).

Let $\mathcal{V}(\Omega, \mathcal{S})$ be defined as follows:

$\Omega=\left\{\omega_{1}, \omega_{2}, \ldots, \omega_{K}\right\}, \quad \mathcal{S}=2^{\Omega}, \quad K \in \mathbb{N}$
Let for each $k \in \mathbb{N}[K] \pi_{k}=\left(\mu_{k}, v_{k}\right) \in \mathcal{V}(\Omega, \mathcal{S})$ has the form:

$\mu_{k}\left(\omega_{i}\right)=v_{k}\left(\omega_{i}\right)= \begin{cases}1 & \text { if } \quad i=k \\ 0 & \text { otherwise }\end{cases}$

We assume that the IV-probability $\mathfrak{P}: \mathcal{V}(\Omega, \mathcal{S}) \rightarrow \mathcal{J}$ has the form $\mathfrak{P}=\mathfrak{P}_{\hat{P}}$, where $\hat{P}$ is the probability defined on $(\Omega, \mathcal{S})$ by the conditions: $\hat{P}\left(\left\{\omega_{k}\right\}\right)=p_{k}>0, k \in \mathbb{N}[K]$, $\sum_{k=1}^{K} p_{k}=1$.

The following example corresponds to the case considered in the de Moivre-Laplace CLT.

Let $K=2$. Let for arbitrary $A \in \mathcal{B}(\mathbb{R})$ observable $x_{j}$ : $\mathcal{B}(\mathbb{R}) \rightarrow \mathcal{V}(\Omega, \mathcal{S}), j \in \mathbb{N}$, be uniquely defined by the conditions

$x_{j}(A)=\left\{\begin{array}{lll}\mathbf{0}_{\mathbf{\Omega}} & \text { if } & A \cap\{0,1\}=\emptyset, \\ \pi_{1} & \text { if } & A \cap\{0,1\}=\{0\}, \\ \pi_{2} & \text { if } & A \cap\{0,1\}=\{1\} .\end{array}\right.$

We assume that the IV-observables $\left\{x_{j}\right\}_{j \in \mathbb{N}}$ are independent. Then for $j \in \mathbb{N}$

$e^{b}=\mathbb{E}^{b}\left(x_{j}\right)=e^{\natural}=\mathbb{E}^{\natural}\left(x_{j}\right)=p_{2}$

and

$\mathbb{D}^{2, b}\left(x_{j}\right)=\mathbb{D}^{2, \natural}\left(x_{j}\right)=p_{1} p_{2}>0, \sigma^{b}=\sigma^{\natural}=\sqrt{p_{1} p_{2}}$.

Thus, by Theorem 12 , for $t \in \mathbb{R}$

$\mathfrak{P}^{b}\left(\frac{\sum_{j=1}^{n} x_{j}-n p_{2}}{\sqrt{n p_{1} p_{2}}}((-\infty, t))\right) \rightarrow \Phi(t)$, as $n \rightarrow \infty$,

$\mathfrak{P}^{\natural}\left(\frac{\sum_{j=1}^{n} x_{j}-n p_{2}}{\sqrt{n p_{1} p_{2}}}((-\infty, t))\right) \rightarrow \Phi(t)$, as $n \rightarrow \infty$.

\subsection{Application of the Lindeberg CLT}

Let $(\mathcal{V}(\Omega, \mathcal{S}), \mathfrak{P})$ be the IV-probability space defined in the previous subsection for $K=3$ and $p_{1}=p_{3}$. Let for each $n \in \mathbb{N} x_{n j}=x_{j}, k_{n}=n$ and for arbitrary $A \in \mathcal{B}(\mathbb{R})$ the observable $x_{j}: \mathcal{B}(\mathbb{R}) \rightarrow \mathcal{V}(\Omega, \mathcal{S}), j=1,2,3, \ldots$, is uniquely defined by the following conditions:

$x_{j}(A)=\left\{\begin{array}{lll}\mathbf{0}_{\Omega} & \text { if } & A \cap \Gamma_{j}=\emptyset, \\ \pi_{1} & \text { if } & A \cap \Gamma_{j}=\left\{-\sqrt{1+\frac{1}{2^{j}}}\right\}, \\ \pi_{2} & \text { if } & A \cap \Gamma_{j}=\{0\}, \\ \pi_{3} & \text { if } & A \cap \Gamma_{j}=\left\{\sqrt{1+\frac{1}{2^{j}}}\right\}\end{array}\right.$, 
where

$\Gamma_{j}=\left\{-\sqrt{1+\frac{1}{2^{j}}}, 0, \sqrt{1+\frac{1}{2^{j}}}\right\}$.

We assume that $\left\{x_{j}\right\}_{j \in \mathbb{N}}$ are independent. Then for each $j \in$ $\mathbb{N}$

$\mathbb{E}_{(n)}^{b}\left(x_{j}\right)=\mathbb{E}_{(n)}^{\natural}\left(x_{j}\right)=0$

and

$\mathbb{D}_{(n)}^{2, b}\left(x_{j}\right)=\mathbb{D}_{(n)}^{2, \natural}\left(x_{j}\right)=2 p_{1}\left(1+\frac{1}{2^{j}}\right)$.

Thus,

$s_{n}^{2, \mathrm{~b}}=s_{n}^{2, \natural}=2 p_{1}\left(n+1-\frac{1}{2^{n}}\right) \rightarrow \infty$, as $n \rightarrow \infty$.

For a fixed $\varepsilon>0$ and sufficiently large $n$

$$
\begin{aligned}
\mathfrak{l}_{n, b}^{x_{n j}}\left(\varepsilon, s_{n}^{b}\right) & =\mathfrak{r}_{n, \natural}^{x_{n j}}\left(\varepsilon, s_{n}^{\natural}\right) \\
& =\mathbb{E}_{(n)}^{b}\left(\left(x_{j}-\mathbb{E}_{(n)}^{b}\left(x_{j}\right)\right)^{2} I_{\left|x_{j}-\mathbb{E}_{(n)}^{b}\left(x_{j}\right)\right|>\varepsilon s_{n}^{b}}\right)=0
\end{aligned}
$$

as well as

$$
\begin{aligned}
\mathfrak{L}_{n}^{b}(\varepsilon) & =\frac{1}{s_{n}^{2, b}} \sum_{j=1}^{k_{n}} \mathfrak{r}_{n, b}^{x_{n j}}\left(\varepsilon, s_{n}^{b}\right) \\
& =\mathfrak{L}_{n}^{\natural}(\varepsilon)=\frac{1}{s_{n}^{2, \natural}} \sum_{j=1}^{k_{n}} \mathfrak{r}_{n, \natural}^{x_{n j}}\left(\varepsilon, s_{n}^{\natural}\right)=0,
\end{aligned}
$$

since the supports of the probability measures $\left(\mathfrak{P}_{(n)}^{b}\right)_{x_{j}}$ and $\left(\mathfrak{P}_{(n)}^{\natural}\right)_{x_{j}}$ are bounded. Consequently,

$\mathfrak{L}_{n}(\varepsilon)=2 \mathfrak{L}_{n}^{b}(\varepsilon) \rightarrow 0$, as $n \rightarrow \infty$.

Therefore, the convergences

$$
\begin{aligned}
& \mathfrak{P}_{(n)}^{b}\left(\frac{\sum_{j=1}^{k_{n}} x_{n j}}{s_{n}^{b}}((-\infty, t))\right) \\
& =\mathfrak{P}^{b}\left(\frac{\sum_{j=1}^{n} x_{j}}{s_{n}^{b}}((-\infty, t))\right) \rightarrow \Phi(t), \text { as } n \rightarrow \infty, \\
& \mathfrak{P}_{(n)}^{\natural}\left(\frac{\sum_{j=1}^{k_{n}} x_{n j}}{s_{n}^{\natural}}((-\infty, t))\right) \\
& =\mathfrak{P}^{\natural}\left(\frac{\sum_{j=1}^{n} x_{j}}{s_{n}^{\natural}}((-\infty, t))\right) \rightarrow \Phi(t), \text { as } n \rightarrow \infty
\end{aligned}
$$

for each $t \in \mathbb{R}$ follow from Theorem 9 .

\subsection{Application of the Lyapunov CLT}

We consider the $\mathcal{V}(\Omega, \mathcal{S})$ specified in the previous example for $K=3$.

For arbitrary $n \in \mathbb{N}$, we denote by $\hat{P}_{n}$ the probability defined on $(\Omega, \mathcal{S})$ by the equalities:

$\hat{P}_{n}\left(\left\{\omega_{1}\right\}\right)=\hat{P}_{n}\left(\left\{\omega_{3}\right\}\right)=\frac{1-4^{-n}}{2}, \quad \hat{P}_{n}\left(\left\{\omega_{2}\right\}\right)=4^{-n}$

and by $\mathfrak{P}_{(n)}$ the IV-probability $\mathfrak{P}_{(n)}: \mathcal{V}(\Omega, \mathcal{S}) \rightarrow \mathcal{J}$ of the form $\mathfrak{P}_{(n)}=\mathfrak{P}_{\hat{P}_{n}}$.

For each $n \in \mathbb{N}$, we assume that $k_{n}=n$ and for $A \in \mathcal{B}(\mathbb{R})$ the IV-observable $x_{n j}: \mathcal{B}(\mathbb{R}) \rightarrow \mathcal{V}(\Omega, \mathcal{S}), j \in \mathbb{N}[n]$, is defined by the formula

$x_{n j}(A)=\left\{\begin{array}{llll}\mathbf{0}_{\mathbf{\Omega}} & \text { if } & & A \cap\{-1,0,1\}=\emptyset, \\ \pi_{1} & \text { if } & A \cap\{-1,0,1\}=\{-1\}, \\ \pi_{2} & \text { if } & A \cap\{-1,0,1\}=\{0\}, \\ \pi_{3} & \text { if } & A \cap\{-1,0,1\}=\{1\} .\end{array}\right.$

We additionally assume that $\left\{x_{n j}\right\}_{j \in \mathbb{N}[n]}$ are independent for each positive integer $n$.

Fix $n \in \mathbb{N}$ and $\delta>0$. For arbitrary $j \in \mathbb{N}[n]$

$$
\begin{aligned}
\mathbb{E}_{(n)}^{b}\left(x_{n j}\right) & =\mathbb{E}_{(n)}^{\natural}\left(x_{n j}\right)=0, \\
\mathbb{E}_{(n)}^{b}\left(\left|x_{n j}\right|^{2+\delta}\right) & =\mathbb{E}_{(n)}^{\natural}\left(\left|x_{n j}\right|^{2+\delta}\right)=1-4^{-n}, \\
\mathbb{D}_{(n)}^{2, b}\left(x_{n j}\right) & =\mathbb{D}_{(n)}^{2, \natural}\left(x_{n j}\right)=\mathbb{E}_{(n)}^{b}\left(\left(x_{n j}\right)^{2}\right) \\
& =\mathbb{E}_{(n)}^{\natural}\left(\left(x_{n j}\right)^{2}\right)=1-4^{-n} .
\end{aligned}
$$

Therefore,

$s_{n}^{b}=s_{n}^{\natural}=\sqrt{n\left(1-4^{-n}\right)}$

and

$$
\begin{aligned}
& \frac{\sum_{j=1}^{k_{n}} \mathbb{E}_{(n)}^{b}\left(\left|x_{n j}-\mathbb{E}_{(n)}^{b}\left(x_{n j}\right)\right|^{2+\delta}\right)}{s_{n}^{2+\delta, b}} \\
& +\frac{\sum_{j=1}^{k_{n}} \mathbb{E}_{(n)}^{\natural}\left(\left|x_{n j}-\mathbb{E}_{(n)}^{\natural}\left(x_{n j}\right)\right|^{2+\delta}\right)}{s_{n}^{2+\delta, \natural}} \\
& =\frac{2 n\left(1-4^{-n}\right)}{n^{1+\frac{\delta}{2}}\left(1-4^{-n}\right)^{1+\frac{\delta}{2}}}=\frac{2}{n^{\frac{\delta}{2}}\left(1-4^{-n}\right)^{\frac{\delta}{2}}} \rightarrow 0,
\end{aligned}
$$

as $n \rightarrow \infty$.

Therefore, by Theorem 10,

$\mathfrak{P}_{(n)}^{b}\left(\frac{\sum_{j=1}^{k_{n}} x_{n j}}{s_{n}^{b}}((-\infty, t))\right) \rightarrow \Phi(t)$, as $n \rightarrow \infty$, 


$$
\mathfrak{P}_{(n)}^{\natural}\left(\frac{\sum_{j=1}^{k_{n}} x_{n j}}{s_{n}^{\natural}}((-\infty, t))\right) \rightarrow \Phi(t), \text { as } n \rightarrow \infty
$$

for $t \in \mathbb{R}$.

\section{Conclusions}

In this paper, we proved the Lindeberg CLT, the Lyapunov CLT, and the Feller theorem for IV-events. The results obtained in the IV-probabilistic case correspond to the classical limit theorems for independent but not necessary identically distributed random variables. We also presented examples of applications of the aforementioned limit theorems for scaled sums of IV-observables. Our future possible considerations will concern further development of the probability theory for IV-events. In our opinion, the theorems proved in this paper can be important tools for future statistical applications.

\section{Compliance with Ethical Standards}

Conflict of interest The authors declare that they have no conflict of interest.

Ethical approval This article does not contain any studies with human participants or animals performed by any of the authors.

Open Access This article is distributed under the terms of the Creative Commons Attribution 4.0 International License (http://creativecomm ons.org/licenses/by/4.0/), which permits unrestricted use, distribution, and reproduction in any medium, provided you give appropriate credit to the original author(s) and the source, provide a link to the Creative Commons license, and indicate if changes were made.

\section{References}

Atanassov K (1999) Intuitionistic fuzzy sets: theory and applications. Physica, New York

Atanassov K (2012) On intuitionistic fuzzy sets theory. studies in fuzziness and soft computing 283. Springer, Berlin

Billingsley P (1986) Probability and measure, 2nd edn. Wiley Press, New York

Birkhoff G, Von Neumann J (1936) The logic of quantum mechanics. Anna Math 37:823-843

Blanco-Fernández A, Casals MR, Colubi A, Corral N, García-Bárzana M, Gil MÁ, González-Rodríguez G, López MT, Lubiano MA, Montenegro M, Ramos-Guajardo AB, la Rosa De, de Sáa S, Sinova B (2014) A distance-based statistical analysis of fuzzy numbervalued data. J Approx Reason 55(1487-1501):1601-1605

Carathéodory C (1956) Mass und integral und ihre algebraisierung. Birkäuser, Boston

Chang CC (1958) Algebraic analysis of many valued logics. Trans Am Math Soc 88:467-490

Ciungu LC, Riečan B (2010) Representation theorem for probabilities on IFS-events. Inf Sci 180:793-798
Cignoli R, D’Ottaviano I, Mundici D (2000) Algebraic foundations of many-valued reasoning. Kluwer Academic Publishers, Dordrecht

Couso I, Dubois D, Sánchez L (2014) Random sets and random fuzzy sets as ill-perceived random variables. Springer briefs in applied sciences and technology, Cham

Deng JL (1989) Introduction to grey system theory. J Grey Syst 1:1-24

Deschrijver G, Kerre EE (2003) On relationship between some extensions of fuzzy set theory. Fuzzy Sets Syst 133:227-235

Dubois D, Gottwald S, Hajek P, Kacprzyk J, Prade H (2005) Terminological difficulties in fuzzy set theory-the case of "Intuitionistic Fuzzy Sets". Fuzzy Sets Syst 156:485-491

Dubois D, Prade H (2012) Gradualness, uncertainty and bipolarity: making sense of fuzzy sets. Fuzzy Sets and Syst 192:3-24

Fine TL (1973) Theories of probability: an examination of foundations. Academic Press, New York

Gil MÁ, Hryniewicz O (2009) Statistics with imprecise data. In: Myers R (ed) Encyclopedia of complexity and system science. Springer, Heidelberg, pp 8679-8690

Grattan-Guiness I (1975) Fuzzy membership mapped onto interval and many-valued quantities. Zeit. Math. Logik. Grundlagen Math. 22:149-160

Grzegorzewski P, Mrówka E (2002) Probability of intuitionistic fuzzy events. In: Grzegorzewski P, Hryniewicz O, Gil MÁ (eds) Soft methods in probability, statistics and data analysis. Physica-Verlag, Heidelberg, pp 105-115

Gudder S (1979) Stochastic methods of quantum mechanics. Elsevier, North-Holland

Jahn KU (1975) Intervall-wertige Mengen. Math. Nachrichten 68:115132

Kruse R, Meyer KD (1987) Statistics with vague data. Reidel Publ. Co., Dordrecht

Kuková M (2011) An extension of a state from interval valued fuzzy sets to MV-Algebra. In: 3rd international workshop Quantum Structures, Kočovce, Slovakia

Kwakernaak H (1978) Fuzzy random variables. part I: definitions and theorems. Inf Sci 15:1-29

Lendelová K (2006) Conditional IF-probability. In: Advances in soft computing: soft methods for integrated uncertainty modelling, $\mathrm{pp}$ 275-283

Lendelová K, Petrovičová J (2006) Representation of IF-probability for MV-algebras. Soft Comput 10:564-566

Liu YK, Liu B (2003) Fuzzy random variables: a scalar expected value operator. Fuzzy Optim Decis Mak 2:143-160

Mundici D (1986) Interpretation of AFC*-algebras in Lukasiewicz sentential calculus. J Funct Anal 65:15-63

Mundici D (2011) Advanced Lukasiewicz calculus and MV-algebras. Springer, New York

Nowak P (2003) Monotone measures of intuitionistic fuzzy sets. In: Proceedings of the 3rd Conference of the European society for Fuzzy logic and technology, EUSFLAT 2003, Zittau, pp 172-176

Nowak P (2004a) Construction of monotone measures of intuitionistic fuzzy sets. In: Atanassov K, Hryniewicz O, Kacprzyk J (eds) Soft Comput Found Theor Asp. Academicka Oficyna Wydawnicza EXIT, Warsaw, pp 303-317

Nowak P (2004b) Integral with respect to a monotone measure on intuitionistic fuzzy sets. In: de Baets B, de Caluwe R, de Tre G, Fodor J, Kacprzyk J, Zadrozny S (eds) Current issues in data and knowledge engineer. Academicka Oficyna Wydawnicza EXIT, Warsaw, pp 378-387

Nowak P, Hryniewicz O (2015) Generalized versions of MV-algebraic central limit theorems. Kybernetika 51:765-783

Nowak P, Hryniewicz O (2016) On generalized versions of central limit theorems for IF-events. Inf Sci 355:299-313

Pták P, Pulmannová S (1989) Kvantové logiky (in Slovak). Veda, Bratislava 
Puri ML, Ralescu DA (1986) Fuzzy random variables. J Math Anal Appl 114:409-422

Renčová M (2010) A generalization of probability theory on MValgebras to IF-events. Fuzzy Sets Syst 161:1726-1739

Riečan B (1999) On limit theorems in fuzzy quantum spaces. Fuzzy Sets Syst 101:79-86

Riečan B (2000) On the probability theory on MV-algebras. Soft Comput 4:49-57

Riečan B (2004) On the law of large numbers on IFS Events. Advances in soft Computing 2. Springer, Dortmundt, Germany, pp 677-680

Riečan B (2006a) On two ways for the probability theory on IF-sets. In: Soft methods for integrated uncertainty modelling, advances in soft computing. Springer, Berlin pp 285-290

Riečan B (2006b) On a problem of Radko Mesiar: general form of IF-probabilities. Fuzzy Sets Syst 152:1485-1490

Riečan B (2007a) M-probability theory on IF-events. In: Štěpnička M, Novák V, Bodenhofer U (eds) Proceedings on EUSFLAT'2007, Universitas Ostraviensis, pp 227-230

Riečan B (2007b) Probability theory on IF events. In: Aguzzoli S et al. (eds) Trends and progress in system identification. Papers in honor of Daniele Mundici on the occasion of his 60th birthday. Lecture notes in computer science 4460. Springer, Berlin, pp 290-308

Riečan B, Král' P (2010) Probability on interval valued events. In: Proceedings of the 11th international workshop on generalized nets and 2nd international workshop on generalized nets, intuitionistic fuzzy sets and knowledge engineering. London, pp 66-70

Riečan B, Mundici D (2002) Probability on MV-algebras. In: Pap E (ed) Handbook of measure theory. Elsevier, Amsterdam, pp 869-909

Riečan B, Neubrunn T (1997) Integral measure and ordering. Kluwer Academic Publishers, Bratislava
Sambuc R (1975) Fonctions $\Phi$-floues. Application a l'aide au diagnostic en pathologie thyroidienne. Dissertation. Faculté de Médecine de Marseille

Samuelčík K, Hollá I (2013) The limit theorems on the interval valued events. In: 9th international workshop on IFSs, Banská Bystrica. Notes on intuitionistic fuzzy sets 19(2):31-41

Szmidt E, Kacprzyk J (1999a) A concept of a probability of an intuitionistic fuzzy event. In: Proceedings of the 1999 IEEE international fuzzy systems conference, Seoul, pp 1346-1349

Szmidt E, Kacprzyk J (1999b) Probability of intuitionistic fuzzy events and their applications in decision making. In: Proceedings of the 9th congreso EUSFLAT'99, pp 457-460

Tripathy BC, Baruah A, Et M, Gungor M (2012) On almost statistical convergence of new type of generalized difference sequence of fuzzy numbers. Iran J Sci Technol Trans A: Sci 36(2):147-155

Tripathy BC, Das PC (2012) On convergence of series of fuzzy real numbers. Kuwait J Sci Eng 39(1A):57-70

Tripathy BC, Ray GC (2012) On mixed fuzzy topological spaces and countability. Soft Comput 16(10):1691-1695

Tripathy BC, Sarma B (2012) On I-convergent double sequences of fuzzy real numbers. Kyungpook Math J 52(2):189-200

Türkşen IB (1986) Interval valued fuzzy sets based on normal forms. Fuzzy Sets Syst 20:191-210

Varadarajan VC (1968) Geom Q Mech. van Nostrand, Princeton, New Jersey

Zadeh LA (1975) The concept of a linguistic variable and its application to approximate reasoning, part I. Inf Sci 8:199-249 